\title{
LA NUEVA FORMA DE HACER NEGOCIOS, UN COMPARATIVO DE LA CULTURA DEL EMPRENDIMIENTO ENTRE MÉXICO Y PERÚ
}

\section{THE NEW WAY OF DOING BUSINESS, A COMPARATIVE OF ENTREPRENEURSHIP CULTURE BETWEEN MEXICO AND PERU}

Angel Benavides Camarillo ${ }^{1}$

\begin{abstract}
RESUMEN
Desde décadas atrás se busca que la acción de emprender se convierta en una cultura en países como México y Perú a tal grado que se han modificado desde los programas de estudio de las escuelas hasta los planes de desarrollo a nivel gobierno, con la finalidad de poder competir y ser reconocidos como impulsores del emprendimiento y ver a este como un elemento clave para el beneficio económico y social que permite a los individuos de un país tener su propia fuente de ingresos y además otorgar trabajo a personas de su mismo ámbito social. Disminuyendo así la tasa de desempleo y haciendo crecer el ingreso monetario de los individuos, en consecuencia, mejora las condiciones sociales del país.

El presente artículo recopila información de la planeación y actividades que se han realizado en los últimos años en los países de México y Perú, cuáles han sido los negocios de nueva creación que han alcanzado renombre por los beneficios que otorgan a sus participantes, haciendo uso principalmente de las tecnologías de la información y comunicación como el caso de las startup. Para Eduardo Morelos, director de Startupbootcamp Fintech México, la startupes una gran empresa en su etapa temprana; a diferencia de una Pyme, aquella se basa en un negocio que será escalable más rápida y fácilmente, haciendo uso de tecnologías digitales.
\end{abstract}

Palabras Claves: Emprendimiento, México, negocios, Perú.

\begin{abstract}
For decades, it has been sought that entrepreneurship becomes a culture in countries such as Mexico and Peru to such an extent that from school study programs to government-level development plans have been modified with the purpose of being able to compete and be recognized as promoters of entrepreneurship and see it as a key element for the economic and social benefit that allows individuals in a country to have their own source of income and in addition to grant work to people of the same social area, decreasing the rate of unemployment and increasing the monetary income of the individuals and consequently the improvement of the social conditions of the country.

This article collects information on the planning and activities that have been carried out in recent years in Mexico and Peru, what have been the newly created businesses that have achieved renown for the benefits they give to their participants, making use mainly of information and communication technologies as in the case of startups. For Eduardo Morelos, director of Startupbootcamp Fintech Mexico, the startupes a large company in its early stage; unlike a SME, that is based on a business that will be scalable faster and easier, making use of digital technologies.
\end{abstract}

Key Words: Entrepreneurship, Mexico, business, Peru.

${ }^{1}$ Universidad Popular Autónoma del Estado de Puebla. Puebla-México. E-mail: angel.benavides@upaep.mx 


\section{INTRODUCCIóN}

Problemática. ¿En qué consiste la cultura del emprendimiento entre México y Perú?

Objetivo. Analizar la cultura del emprendimiento, estableciendo sus caracteristicas más relevantes en México y Perí.

Metodología. Investigación cualitativa. Por ser un trabajo descriptivo, no requiere hipótesis.

Justificación. El emprendimiento es parte del quehacer diario de varios millones de personas en países latinoamericanos. Conocer sus antecedentes y más aún, la estadística que ayude a dimensionar los puntos de convergencia entre los emprendedores de Perú y México permitirá tener una base de datos importante en la búsqueda posterior de información específica sobre el tema. Por ello, el presente estudio se basa en el análisis de bibliografia actualizada desde un enfoque comparativo.

Si bien la metodología aplicada permite alcanzar el objetivo principal — posibilitando una explicación directa de los principales elementos a comparar entre la cultura del emprendimiento-, esta investigación se ve limitada en la obtención de información por la exigencia de fuentes actuales, Internet, revistas y libros con información reciente sobre emprendedores en México y Perú.

No es fácil determinar con exactitud cuándo y dónde surgen los primeros emprendedores en los dos países ya citados. Brenes \& Jerry (2012), en su libro The future of entrepreneurship in Latín América, señalan que son las fuerzas externas ${ }^{2}$ quienes ejercen mayor presión en una región o país y crean las condiciones propicias para que las personas inicien con un proyecto que consideran puede crear beneficios económicos a corto, mediano y largo plazo.

En el gráfico Tasa de desocupación de México y Perú entre 1995 y 2010, se puede observar que en un periodo de más de diez afios, se incrementó hasta cinco veces la cantidad de desempleados en Perú comparándola con México. Brenes \& Jerry (2012) hacen

\footnotetext{
${ }^{2}$ Estas fuerzas externas se dan a través de los cambios macroeconómicos y microeconómicos, los efectos de la globalización en ramas como la tecnologia, la cultura, la migración, la participación directa de la mujer en la economia del hogar, políticas públicas implementadas por los gobiernos, crecimiento poblacional, corrupción, desastres naturales, tasa de desocupación, entre otros.
}

referencia, además, al momento en que la cantidad de emprendedores se incrementa debido a las condiciones económicas y sociales que se presentan en una sociedad. Coincidentemente al inicio de la década del 90 los dos países presentaron problemas por la apertura de las fronteras y se dio paso a un modelo económico neoliberal.

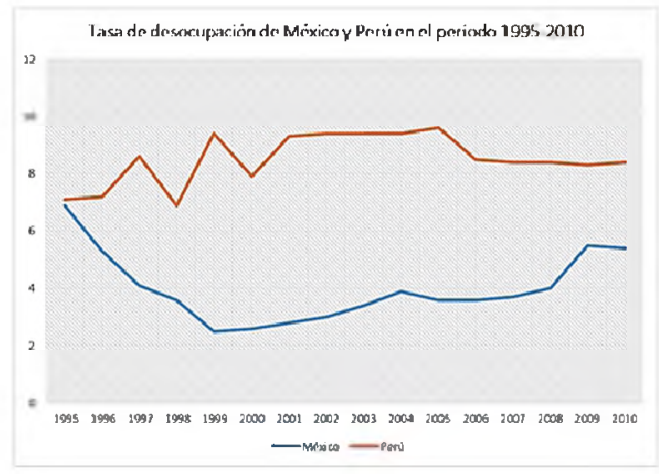

Figura 1. Tasa de desocupaciớn de México y Perú entre 1995 y 2010

Fuente: Elaboración propia a partir de datos del documento Evolución Socioeconómica del Perú, del Centro Nacional de Planeamiento Estratégico. (1990-2010)

En este ensayo se compara, con datos actuales, los beneficios de implementar un sistema de emprendimiento así como los resultados que este genera en dos países con características en común; lo cual resulta relevante pues desde un punto de vista de trabajo colaborativo entre gobiernos, universidades y particulares permite compartir las experiencias de éxito o fracaso de los emprendedores mexicanos y peruanos.

\section{DESARROLLOTEMÁTICO}

\section{Cultura del emprendimiento}

Aprincipios del siglo XVII se comienza a dar un fuerte movimiento entre dos de los principales puertos del norte y sur de América. El puerto de Acapulco, Guerrero en México y el puerto del Callao, Lima en Perú; ambos situados en la costa del Pacífico, como lo refiere Suárez (2015):

El comercio con México era una prolongación del comercio del Perú con Panamá y Guatemala; los navios viajaban al norte con pasas, conservas, aceitunas, mercurio y, sobre todo, cacao, vino, 
vinagre y plata, y regresaban con mereadentas asiáticas y europeas obtenidas en la ciudad de México, y con productos de la tierra (brea, madera, affil, bálsamo y palo de Campeche).

Al formar parte de los países de la corona espafola los dos reinos - México y Perúeran factor clave para realizar intercambios comerciales, incluso desde antes de la llegada de los espafioles se cree que ya existía una ruta comercial entre estas dos regiones teniendo como evidencia vestigios de conchas $y$ cerámicas, que eran distintivas de lugares muy específicos y que se encontraban en dos regiones tan lejanas como Cuzco (Perú) y Tenochtitlan (México): dos de los centros religiosos, sociales y comerciales más importantes del continente americano hace más de 800 años (Schávelzon, 1979).

Han pasado varios siglos y los actos de comercio no han terminado. Ambos países se caracterizan por un espíritu emprendedor entre sus habitantes, que los alienta a buscar la forma de iniciar un negocio y generar un ingreso económico que satisfaga, en un principio, sus necesidades primarias y merced a un crecimiento ordenado y fruto del esfuerzo, les permita generar una calidad de vida.

No obstante, en los últimos años, el espíritu emprendedor ha disminuido en México, tal como lo muestra la figura 1. Esto quizá se deba al hartazgo de la ciudadania, provocado por los malos manejos del gobierno en los programas que se orientan hacia el emprendimiento y la apertura a varias empresas transnacionales que otorgan un "bienestar" económico y una estabilidad laboral.

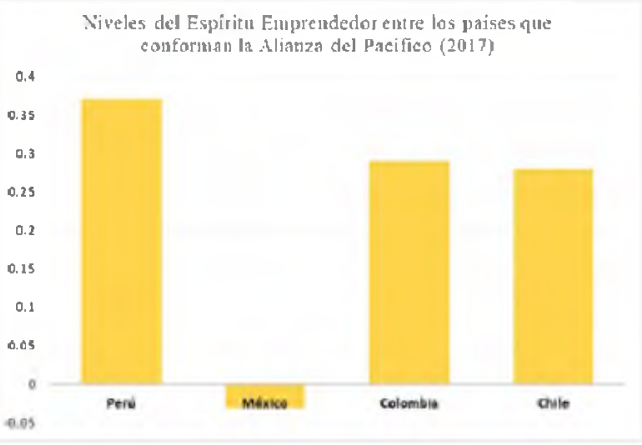

Figura 2. Niveles del espíritu emprendedor entre los países de la Alianza del Pacífico (2017).

Fuente: Elaboración propia con información del Global Entrepreneurship Monitor 2017.

La siguiente tabla nos permite hacer un análisis del comportamiento del emprendedor mexicano en el mismo año 2017. Una de las posibles causas de disminución del espíritu emprendedor en México, en comparación con Perú, puede encontrarse en la siguiente estadística: el $75 \%$ de las empresas cierra antes de terminar el segundo afo y el $89 \%$ inicia un negocio con dinero propio o de la familia (López, 2016); el apalancamiento financiero aún no es un tema visto favorablemente por los emprendedores. Solo con esta estadística, de porcentajes muy elevados, se puede concluir que si el emprendedor no cuenta con dinero de sus ahorros se le complica iniciar su negocio propio.

Revisando la última fila de la tabla: Cifras sobre el emprendimiento en México (2017), se analiza el miedo al fracaso que experimentan los emprendedores mexicanos en un 33\%. Este temor a la pérdida de su inversión se justifica en que una tercera parte de las empresas cierra en el segundo afio, de no tener una correcta planeación; de allí que los propietarios compartan la idea de que los primeros meses para cualquier micro empresa son los más dificiles. 
Tabla 1

Cifras sobre el emprendimiento en México (2017).

$33 \%$ De los emprendedores en México tienen entre 25 y 34 años.

$54 \%$ De los que hoy arrancan un negocio son solteros.

$66 \%$ De los emprendedores son hombres vs el $34 \%$ mujeres.

$75 \%$ De las empresas cierran antes de terminar el segundo año.

$14 \%$ De las empresas nuevas obtiene ingresos fuera del mercado nacional.

$52 \%$ De los negocios son operados desde casa / sin oficina.

89\% Arrancaron con dinero propio o de familia vs el 3\% que recibieron inversión formal.

$66 \%$ Vende menos de $\$ 500,000.00$ pesos al año y el $80 \%$ vende menos de $\$ 1$ millón al año.

$30 \%$ Arrancaron su negocio por necesidad. El resto por buscar un mejor estilo de vida $y$ un crecimiento.

33\% De los mexicanos que quieren emprender, no lo hacen por miedo al fracaso.

Fuente: Miguel Senderos. Entrepreneur México. 29 de marzo, 2017 (Senderos, 2017).

En el año 2013 surge en México, el Instituto Nacional del Emprendedor (INADEM); el cual busca interactuar con el Sistema Educativo Nacional diseñando metodologías específicas en los programas escolares, a fin de lograr una cultura de innovación (Faesler, 2013). El concepto del INADEM es formar creadores de empleo; sin embargo, esta política pública tiene antecedentes que lamentablemente no han dado buenos resultados, entre estos se encuentra el impulso fallido a las MiPyMes (Micro, Pequeñas y Medianas Empresas) que realizó el entonces presidente de México, Dr. Ernesto Cedillo Ponce de León; posteriormente el Lic. Vicente Fox Quesada con su plan de "changarros" y el presidente Felipe Calderón Hinojosa con su programa de talleres (Lascuraín, 2014).

Las políticas públicas que se han diseñado con el objetivo de formar creadores de empleo no han sido del todo eficaces, puesto que no han alcanzado las metas propuestas. El Dr. Aguilar Villanueva, citado en la obra de Franco Corzo (2013), sostiene que el éxito de una política pública radica en su orientación hacia objetivos

${ }^{3}$ Changarro. En México tienda pequeña o tendejón. (Diccionario de la Lengua Española). de interés o beneficio público y su idoneidad para realizarlos; asimismo, debe promover la participación ciudadana con el gobierno en la definición de objetivos, instrumentos y acciones de la política.

Uno de los intereses principales a nivel legislativo es impulsar el desarrollo económico de una región. Este es el punto de partida para la generación de políticas públicas diseñadas para incrementar el número de emprendedores y que estos se conviertan en empresarios exitosos, en un lapso de tiempo mediano o largo, aumentando la oferta laboral y otorgando un beneficio económico-social al país.

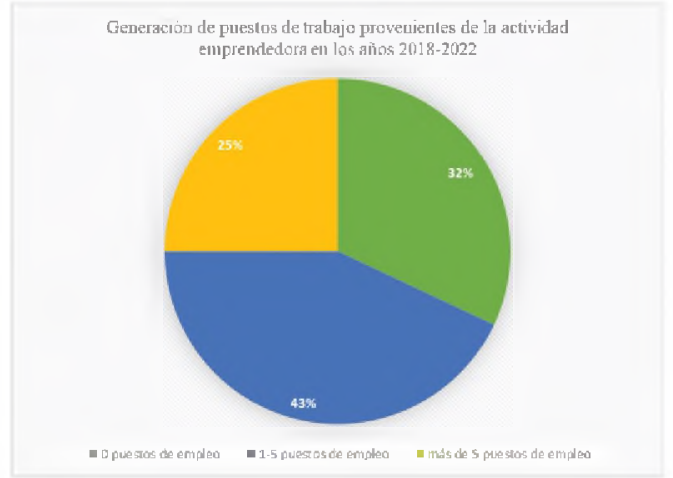

Figura 3. Generación de puestos de trabajo a partir de la actividad emprendedora (20182022).

Fuente: Elaboración propia con datos del periódico digital "El comercio" de Perú (Redacción, 2007).

E1 Perú cuenta con la plataforma digital "Emprendedor peruano", que se encarga de otorgar servicios de desarrollo empresarial a través de la implementación de mecanismos e instrumentos para el desarrollo y fortalecimiento empresarial en beneficio del emprendedor peruano, su familia, su región y el país. Esta plataforma está afiliada al Ministerio de la Producción, con el objetivo de promover la competitividad de las micro, pequeñas y medianas empresas (Emprendedor Peruano, 2018).

La pregunta que los departamentos encargados deberían realizarse es si efectivamente existe una mejora en la calidad de vida de familias conformadas por uno, dos o más emprendedores. Posiblemente el resultado coincida con algunas de las estadísticas que se presentan en esta investigación; por ello, es 
necesario evaluar el desarrollo de los programas a los cuales se destina buena parte del recurso monetario, humano y material para hacer realidad la cultura del emprendimiento. Han transcurrido décadas - para ser más exactos, 24 años en México y Perú-, y aún no existen cifras precisas que determinen el impacto económico de la actividad emprendedora, a pesar de contar con una estadística de años atrás que no se supo aprovechar ni a la cual se dio continuidad. Lamentablemente la situación de millones de mexicanos y peruanos no ha llegado a mejorar, aun cuando se habla de una segunda generación: hijos de emprendedores que buscan mejorar sus condiciones de vida.

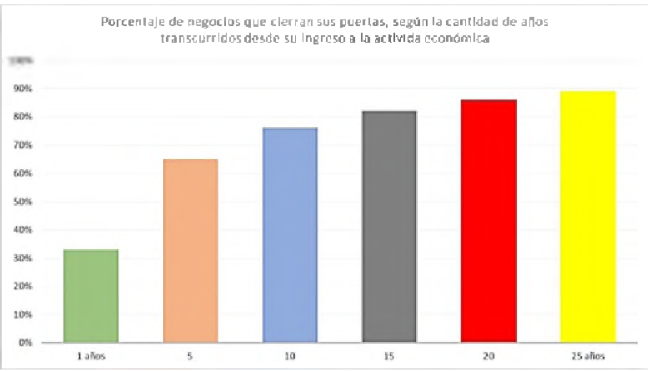

Figura 4. Porcentaje de negocios que cierran sus puertas.

Fuente: Elaboración propia con datos del INEGI. México (Censos económicos 1989, 1994, 1999, 2004, 2009 y 2014).

Semejanzas y diferencias de la cultura de emprendimiento entre México y Perú

En su edición del año 2015, la revista electrónica Gestión señala que el porcentaje de emprendedores de Perú dispuesto a invertir en un nuevo negocio - aun con experiencias negativas al comenzar algún negocio anterior-, es de 50.6\%. En tanto que, en México, las personas mayores de edad que piensan en iniciar un proyecto empresarial en los próximos tres años está reducido a un 19\%.

Contar con un buen crecimiento económico, una movilidad de dinero, un tipo de cambio equilibrado, además de otros indicadores macroeconómicos como baja inflación, generan confianza en aquellos individuos que desean formar parte de la red de emprendedores del Perú. Por otro lado, en el contexto mexicano, quizá sean los resultados de reformas económicas fallidas y el clima de incertidumbre que se genera frente a sus próximas elecciones presidenciales los que provocan desconfianza en quienes desean iniciar un negocio propio en los siguientes tres años.

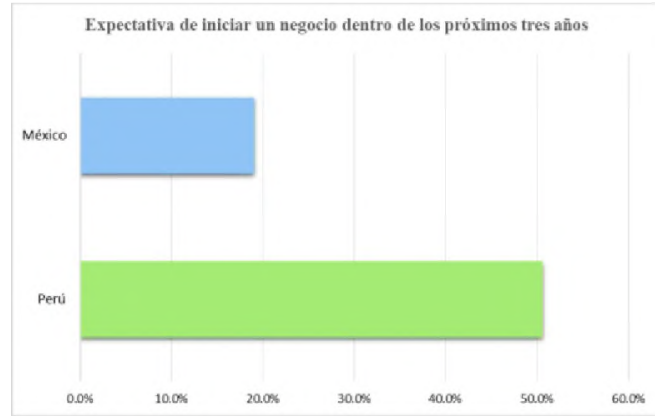

Figura 5. Expectativa de iniciar un negocio en el corto plazo.

Fuente: Elaboración propia con datos de la revista electrónica Gestión 2015.

Aunque en México se trabaje desde hace varias décadas en fomentar la cultura del emprendimiento, las estadísticas indican que aún existe un elevado porcentaje de temor al fracaso; 10 que unido a factores socioeconómicos, políticos y hasta culturales obstaculizan el crecimiento de aquellos emprendedores que desean convertirse en dueños de su propio negocio. En México, las estadísticas indican un mayor temor al fracaso debido a la situación económica que se dio en el país durante el año 2017; donde la mayoría de las reformas en sectores como el energético, salud, educación, fiscales y laborales tuvieron resultados que no fueron percibidos como positivos por la sociedad.

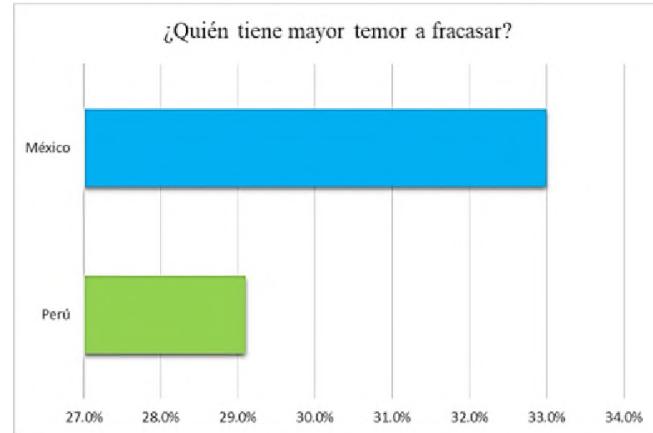

Figura 6. Índice del temor al fracaso. Fuente: Elaboración propia con información de la revista electrónica Gestión 2015. 
El incremento del grado de optimismo en los emprendedores peruanos se puede justificar en los siguientes aspectos:

1. Conocimiento y enseñanza de la cultura del emprendimiento desde los primeros affos de educación en las escuelas públicas y privadas a cargo del Ministerio de la Producción (http://www.produce.gob.pe/).

2. Las instituciones encargadas de facilitar el acceso a los programas de gobierno realizan su labor de manera transparente, objetiva y profesional.

3. Los centros de apoyo al emprendedor se han descentralizado, tomando en cuenta las necesidades de las distintas regiones o zonas donde se encuentran los emprendedores peruanos y diversificando sus programas según los factores de producción de cada comunidad.

4. Traslado de oficinas y centros de apoyo al emprendedor hasta las regiones más alejadas, considerando que el acceso a las herramientas y la capacitación deberán realizarse in situ.

5. Uso de TIC para que el emprendedor pueda recibir capacitación e información en tiempo real, utilizando una computadora y conexión a Internet. Este es el caso de la plataforma Emprendedor Peruano (http://www.emprendedorperuano.pe).

En este contexto, actualmente son más los jovenes quienes al finalizar sus estudios de secundaria o pregrado optan por un negocio propio en lugar de buscar un empleo en alguna empresa. Están comprometidos con generar fuentes de trabajo, y son conscientes de que iniciarse de forma práctica en esta cultura del emprendimiento, requiere mucho esfuerzo fisico e intelectual; pero que, finalmente, les depara una enorme satisfacción en lo personal motivándolos a ser mejores personas.

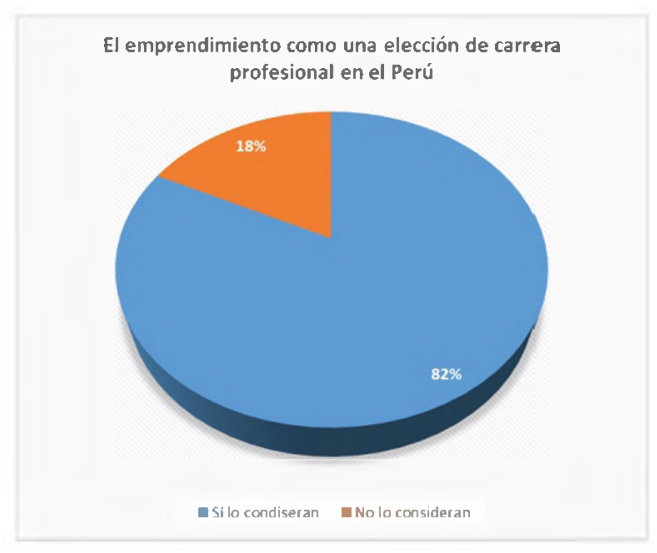

Figura 7. Elección del emprendimiento como una carrera profesional. Estudio realizado por redacción de la revista Gestión de Perú (2015) sobre una población entre 18 a 64 años.

Los habitantes de un país que tengan talento, capital y creatividad tienen la llave del emprendimiento. De acuerdo a Lascuraín (2014) se debe empezar por crear las propias realidades del emprendimiento y no esperar a que alguien más lo haga. Lamentablemente una de estas realidades se convierte en pesadilla al momento de querer iniciar un negocio, la falta de capital es uno de los impedimentos más comunes a los que se enfrentan los emprendedores en México. Por otro lado, el talento y la creatividad son propios de los millones de jóvenes emprendedores que inician con un proyecto que, si es bien gestionado, puede convertirse en el mediano y largo plazo en un referente de mercado.

En México, el Instituto Nacional de Emprendedor (INADEM) ha surgido como un organismo que se encarga de planear el desarrollo de nuevas empresas; asimismo se ha convertido en un puente entre el gobierno y los emprendedores, llevando una dinámica actual a través del uso de las TIC a fin de generar un mayor impacto en emprendedores potenciales. Esto se debe al surgimiento de mecanismos y programas de vanguardia que pretenden la generación de nuevos emprendedores que entiendan la cultura y la situación sociopolítica y económica, tanto como los cambios naturales que se van dando en el pais. 


\section{Retos y oportunidades}

Las empresas que han surgido a partir de una idea y cuyos dueños actuales siguen siendo emprendedores apoyan a micro empresarios para que inicien sus propios proyectos. Esto se realiza a través de pláticas motivacionales y testimonios de éxito con el fin de incentivarlos a que lo intenten cuantas veces sea necesario.

En México y Perú las instituciones de educación básica, media y superior ya se encuentran trabajando en proyectos de acompañamiento del estudiante para la creación de micro empresas de alto impacto.

Lascuraín (2014) señala varios de los retos que los emprendedores enfrentan:

1. Acotamiento del sueño emprendedor. El emprendimiento no es para todos, por tal motivo la palabra emprendedor tiene un gran peso y no cualquiera puede llamarse por sí mismo emprendedor. Todo lo que rodea al emprendimiento, como talleres, apoyos (de todo tipo), escuelas, organizaciones, etc., debe estar regulado y tener un límite para no generar polémica. El emprendimiento no es una receta que se sigue detenidamente y genera por sí solo excelentes resultados.

2. Desarrollar confianza y apoyo. Debe existir seguridad y empatía entre las instituciones y particulares al momento de iniciar un proyecto. La confianza se desarrolla a través de procesos que sean transparentes y se encuentren regulados por instituciones serias.

3. Profesionalizar el emprendimiento. Que el emprendedor no sea el individuo que sobresale por su trabajo diario o aportar una idea nueva; debe ser un profesional en su área, un experto en el tema, con profundo conocimiento en las áreas financieras, mercadotecnia, producción, compras, impuestos y demás. La mayoría de los emprendedores no tiene estos conocimientos de manera innata; sino, fueron aprendiéndolos con el paso de los años.

4. Hacer transparentes los mecanismos de otorgamiento de capital financiero a los emprendedores para no generar desconfianza en la sociedad. En México, según refiere la revista Expansión en Alianza con CNN (2017), durante los años 2015 y 2016, el Instituto Nacional del Emprendedor otorgó recursos a 495,293 emprendedores. Del total de proyectos solventados 9,000 no han demostrado en qué utilizaron el dinero, no acreditaron el cumplimiento de objetivos ni han informado si recibieron las aportaciones privadas o estatales complementarias que requería su solicitud de apoyo.

\section{CONCLUSIONES}

La cultura del emprendimiento en México y Perú es un fenómeno que se vive desde varias décadas atrás, en donde los individuos con visión, actitud, creatividad y con mucho esfuerzo buscan la mejora en sus ingresos económicos logrando satisfacer, con la oferta de sus bienes, las necesidades de un mercado que requiere de productos y servicios con características muy específicas.

El idioma, las creencias, la gastronomía, la música y la cultura en general son elementos que forman a emprendedores de Perú y de México; quienes tienen por objetivo principal iniciar un negocio que en el futuro se convierta en un proyecto de vida generando un ingreso monetario, que además brinde apoyo a sus colaboradores para que estos se conviertan en nuevos emprendedores. Si esto se consigue, se formará una sólida red de profesionistas y expertos en la gestión de micro y pequeñas empresas.

Las TIC se han convertido en la principal herramienta que utilizan los emprendedores en la actualidad. A través de redes sociales y aplicaciones, los productos y servicios llegan a un mayor número de clientes potenciales $\mathrm{y}$ casi siempre, este uso no tiene un costo adicional.

El acceso a una mayor cantidad y calidad de información, así como el apoyo de programas nacionales e internacionales, le brinda al emprendedor los elementos detonantes para que pueda concretizar su idea de negocio y llegue a alcanzar el éxito.

Uno de los principales retos que presentan los emprendedores de ambos países es acceder a la 
capacitación necesaria para dotar de valor agregado a los productos o servicios que ofrecen; así también buscar nuevos mercados meta, que les permita ofrecer sus bienes sin que se desvaloricen en el mercado por la introducción de productos o servicios de otros emprendedores.

Los emprendedores de ambos países atraviesan por problemas similares: la inseguridad, el temor a que su proyecto no funcione, el desconocimiento de programas de apoyo, el poco capital monetario para iniciar con su proyecto, la saturación del mercado, no tener en claro cuál es su mercado meta, entre otros aspectos. No obstante, aun con todas estas adversidades, han enfocado sus esfuerzos en conocer las necesidades reales de sus clientes y colocar bienes que son bien recibidos por los demandantes; gracias a ello, son varios los casos de éxito que se registran cada año. $\mathrm{La}$ actitud de servicio, la pasión al realizar su trabajo, productos de calidad, administración del cliente, entre otros, son los elementos clave para el éxito del emprendedor.

\section{REFERENCIAS BIBLIOGRÁFICAS}

Brenes, E. R., \& Jerry, H. (2012). The future of Entrepreneurship in Latin America. New York: Macmillan.

Emprendedor Peruano. (2018). Obtenido de e mprendedorperuano.pe: http://www.emprendedorperuano.pe/ acerca-de.html

Expansión en Alianza con CNN. (2017). Expansion, México. Obtenido de 9,000 Emprendedores no han comprobado el uso de recursos ante I $\mathrm{N} \quad \mathrm{A} \quad \mathrm{D} \quad \mathrm{E} \quad \mathrm{M}$ : https://expansion.mx/emprendedores/ 2017/06/25/9-000-emprendedoresno-han-comprobado-el-uso-de-

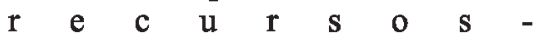
inadem?internal_source $=$ PLAYLIST

Faesler, J. (2013). El Instituto Nacional del Emprendedor. Obtenido de Excelsior: http://www.excelsior.com.mx/opinio n/2013/01/26/julio-faesler/881184

Franco Corzo, J. (2013). Diseño de Políticas Públicas 2da Edición. México: IEXE Editores.
Lascuraín, P. G. (2014). Emprendimiento: ventajas y desafíos de México en Latam. Obtenido de Forbes México: https://www.forbes.com.mx/emprend imiento-ventajas-y-desafios-demexico-en-latam/

Redacción. (2017). El comercio de Perú. Obtenido de elcomercio.pe: https://elcomercio.pe/economia/peruocupa-cuarto-lugar-emprendimientolatinoamerica-1-422604

Redacción Gestión. (2015). Emprendimiento en el Perú se origina más por oportunidad de negocio que por desempleo. Obtenido de Revista Electrónica Gestión Perú: https://gestion.pe/economia/emprendi miento-peru-origina-oportunidadnegocio-desempleo-80578

Schávelzon, D. (1979). Rutas de Intercambio en Mesoamérica y Norte de México, tomo I. XVI Reunión de Mesa Redonda (págs. 75-79). Saltillo, Coahuila, México: UNAM.

Senderos, M. (2017). 10 datos curiosos del emprendimiento en México que debes saber. Entrepreneur. Obtenido de E $n$ n $\quad r$ e $p \quad r$ e $n$ e $u$ r : https://www.entrepreneur.com/article /292067

Suárez, M. (2015). Sedas, Rasos y damascos: Lima y el cierre del comercio triangular con México y Manila en la primera mitad del siglo XVII. América Latina en la historia económica, 22(2), 101-134. Obtenido de scielo.org.mx: http://www.scielo.org.mx/scielo.php? script $=$ sci arttext\&pid $=\mathrm{S} 1405$ $22532015000200005 \& \operatorname{lng}=\mathrm{es} \& \operatorname{lng}=$ es.

Evolución Socioeconómica del Perú (19902010). Centro Nacional de Planteamiento Estratégico. CECOSAMI. 2011, Perú 